\title{
Recombinant expression systems: the obstacle to helminth vaccines?
}

\author{
Peter Geldhof, Veerle De Maere, Jozef Vercruysse and Edwin Claerebout
}

Department of Virology, Parasitology and Immunology, Faculty of Veterinary Medicine, Ghent University, Salisburylaan, 133, B-9820 Merelbeke, Belgium

The need for alternative ways to control helminth parasites has in recent years led to a boost in vaccination experiments with recombinant antigens. Despite the use of different expression systems, only a few recombinants induced high levels of protection against helminths. This is often attributed to the limitations of the current expression systems. Therefore, the need for new systems that can modify and glycosylate the expressed antigens has been advocated. However, analysis of over 100 published vaccine trials with recombinant helminth antigens indicates that it is often not known whether the native parasite antigen itself can induce protection or, if it does, which epitopes are important. This information is vital for a wellthought-out strategy for recombinant production. So, in addition to testing more expression systems, it should be considered that prior evaluation and characterization of the native antigens might help the development of recombinant vaccines against helminths in the long term.

The need for recombinant vaccines against helminths Vaccination against helminths represents a promising alternative for anthelmintic treatment. Even before anthelmintic resistance became a real issue, research started on the development of vaccines because they would be easy to use, would stimulate natural immunity and would be safer in terms of ecotoxicity and presence of residues in meat and milk. Theoretically, several types of vaccine could be used, including vaccines based on: live attenuated material, whole material of dead organisms, (semi-) purified native antigens and recombinant antigens. However, in the case of helminths, there are many practical problems associated with the use of vaccines based on native material. Most importantly, it is very difficult to obtain large quantities of worm material or native antigens from most helminths. An additional problem with native vaccines is the necessity to control for batch differences or to obtain a commercially stable formulation of native parasite material. For these reasons, commercialization will depend on the use of recombinant antigens [1].

\section{Current status on recombinant vaccines}

Since the early 1990 s, more than 100 trials with over 80 different recombinant antigens from 22 different

Corresponding author: Geldhof, P. (peter.geldhof@ugent.be). helminth species have been published (Table 1). Effective recombinant vaccines have been developed for the cestode parasites Taenia ovis, T. saginata, T. solium and Echinococcus granulosus [2]. Apart from these examples, few other recombinants have been produced that induce enough protection to even consider commercialization. Often, the reason for failure has been attributed to the lack of appropriate expression systems to produce these helminth antigens.

About 70 different antigens from 21 different species have been expressed in the Escherichia coli expression system. The outcome of the vaccination trials with these bacterial recombinants showed considerable variation. In taeniid cestodes, very high percentages of protection were obtained in different species, with different antigens and in different hosts (see Table 1). E. coli expressed antigens of trematodes and nematodes have had more variable successes, ranging from $100 \%$ reduction in egg counts in mice and sheep for a Schistosoma mansoni antigen tested against Fasciola hepatica, to 0\% reduction for nematode antigens of Haemonchus contortus and Ostertagia ostertagi (Table 1).

Yeast has been used to express 12 different antigens from six helminth species. The levels of protection obtained with these recombinants vary considerably, although all of them appear to induce some level of protection (see Table 1). The best results were obtained with the recombinant Sh28GST antigen from S. haematobium and the AcAPR-1 antigen from the hookworm Ancylostoma caninum: vaccination with these antigens reduced the egg output by $77 \%$ and $85 \%$, respectively.

The baculovirus expression system has been used to produce eight different antigens from five species (Table 1). A recombinant version of a cathepsin L from $F$. hepatica reduced the worm burden by $52 \%$, and an $S$. japonicum recombinant $\mathrm{SjFABP}$ reduced the parasite burden by $49 \%$. Protection studies with the antigens from nematodes were less successful. Only the Ac-ASP2 antigen from A. caninum reduced the egg output by $69 \%$. All other antigens from the nematode species tested were not protective at all.

Recently, for the first time, the free-living nematode Caenorhabditis elegans was used to express a helminth antigen in sufficient amounts for a vaccine study [3]. This was done for a cathepsin L from $H$. contortus. However, despite the high levels of specific antibody titres, vaccination of sheep did not protect them against a homologous challenge infection. 


\section{Effect of the expression system on protection}

Production of the recombinant antigen in an immunologically active form is the key step in vaccine production. In the main, three different expression systems have been used, that is bacterial, yeast and baculovirus. The advantages and disadvantages of each system have been discussed in detail in Ref. [4] and are summarized in Box 1. The bacterial expression system has been by far the most popular choice to express helminth antigens. However, except for the results with cestodes, the levels of protection induced with $E$. coli recombinants have been rather disappointing. The failure to induce protection has often been

Table 1. Overview of vaccination trials with recombinant helminth antigens ${ }^{a}$

\begin{tabular}{|c|c|c|c|c|c|}
\hline Species & Antigen & Host (administration/adjuvant) & \multicolumn{2}{|c|}{ Reduction (\%) } & Refs \\
\hline \multirow[t]{3}{*}{ Taenia ovis } & To45W (L) & Sheep (i.m./saponin) & & $>90$ & {$[2,19]$} \\
\hline & To16K (L) & Sheep (s.c./saponin) & & 92 & {$[2,19]$} \\
\hline & To18K (L) & Sheep (s.c./saponin) & & 99 & {$[2,19]$} \\
\hline \multirow[t]{3}{*}{ Taenia solium } & TSOL18 (L) & Pigs (i.m./QuilA) & & 100 & {$[2,19]$} \\
\hline & $+16 \mathrm{~K}-\mathrm{GST}$ & & & & {$[20]$} \\
\hline & $+18 \mathrm{~K}-\mathrm{GST}$ & & & & {$[20]$} \\
\hline \multirow[t]{2}{*}{ Taenia saginata } & To45W (L) & Cattle & & 0 & {$[2,19]$} \\
\hline & TSA-18+TSA-9 (L) & Cattle (i.m./QuilA) & & $>90$ & {$[2,19]$} \\
\hline \multirow[t]{2}{*}{ Echinococcus granulosus } & EG95 (L) & Sheep (s.c./QuilA) & & $96-98$ & {$[2,19]$} \\
\hline & & Sheep (s.c./QuilA) & & $96-100$ & {$[2,19]$} \\
\hline \multicolumn{6}{|l|}{ Trematodes } \\
\hline \multirow[t]{7}{*}{ Fasciola hepatica } & Fh15FABP $(I) *$ & Rabbits (s.c./FCA) & $43-76$ & n.t. & [21] \\
\hline & & Sheep (s.c./FCA) & 0 & n.t. & [21] \\
\hline & Sm14 (F/I) & Rabbits (s.c./FCA) & 89 & n.t. & {$[21]$} \\
\hline & & Sheep (s.c./MPL-RIBI + alum) & 100 & n.t. & [21] \\
\hline & & Mice (s.c./FCA) & 100 & n.t. & [21] \\
\hline & rSbGST $(F)^{*}$ & Cattle (i.m./alum, QuilA, FCA) & 0 & n.t. & [21] \\
\hline & Saposine 2 (I) & Rabbits (s.c./TiterMax) & 81 & $73-84$ & {$[21]$} \\
\hline \multirow[t]{3}{*}{ Fasciola gigantica } & rFgFABP $(\mathrm{I})^{*}$ & Cattle (i.m./FCA) & 1 & n.t. & {$[21]$} \\
\hline & & Cattle (i.m./QuilA) & 11 & n.t. & {$[21]$} \\
\hline & & Buffaloes (s.c./FCA) & 36 & 98 & {$[21]$} \\
\hline \multirow[t]{2}{*}{ Schistosoma mansoni } & Sm14 (F/l) & Mice (s.c./RIBI) & $43-67$ & n.t. & {$[22]$} \\
\hline & Paramyosin (I)* & Mice (i.d./BCG) & 33 & n.t. & [23] \\
\hline \multirow{8}{*}{ Schistosoma japonicum } & SjFABP (I) & Rats (i.d./FCA) & 32 & n.t. & [9] \\
\hline & & Sheep (i.m./FCA) & 59 & $23-70$ & [9] \\
\hline & & Mice (s.c./FCA) & 33 & 47 & [9] \\
\hline & SVLBP (F) & Cattle (i.m./FCA) & 30 & $53-89$ & [9] \\
\hline & Sjc26GST (F) & Mice (s.c./FCA) & 23 & 59 & [9] \\
\hline & & Pigs (i.d./Alum) & n.t. & 53 & [9] \\
\hline & & Sheep (i.m./FCA) & 62 & 38 & [9] \\
\hline & $\mathrm{Sj} 23(\mathrm{I})$ & Sheep (i.m./FCA) & $58-66$ & $35-58$ & [9] \\
\hline Schistosoma bovis & Sb28GST $(F)^{*}$ & Sheep (s.c./FCA) & 37 & 18 & {$[26]$} \\
\hline & & Goats (s.c./FCA) & 46 & 35 & {$[27]$} \\
\hline & & $\begin{array}{l}\text { Calves (i.m./FCA/ } \\
\text { infection with S. mattheei) }\end{array}$ & 50 & 89 & [1] \\
\hline & Fh15FABP (I) & Mice (s.c./FCA) & 72 & n.t. & {$[28]$} \\
\hline Nematodes & & & & & \\
\hline Ancylostoma ceylanicum & AceES-2 (F) & Hamsters (s.c./Alum) & n.t. & n.t. & [29] \\
\hline Ancylostoma caninum & Ac-ASP1 (F) & Mice (i.p./Alum) & 60 & 0 & [29] \\
\hline & $A c-A P(F)$ & Dogs (s.c./Cal) & $5-35$ & 0 & {$[29]$} \\
\hline & Ac-TMP (F) & Dogs (s.c./Alum) & 11 & 0 & {$[29]$} \\
\hline & Ac-MTP-1 (F) & Dogs (i.m./AS02) & 0 & 0 & {$[29]$} \\
\hline
\end{tabular}


Table 1 (Continued)

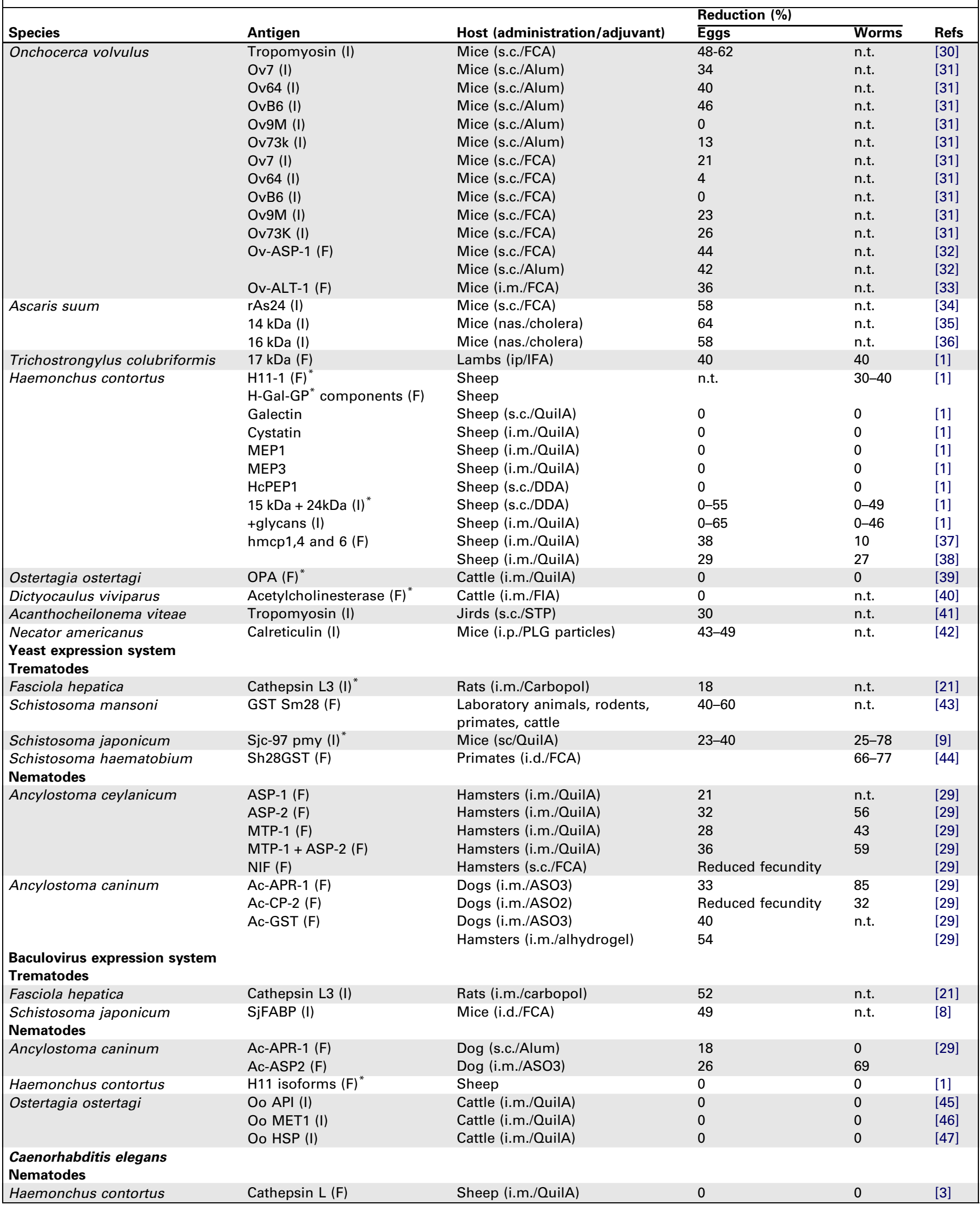

${ }^{a}$ NB This table is not comprehensive.

Abbreviations: n.t.: not tested; i.m.: intramuscular; i.d: intradermal; s.c.: subcutaneous; nas.: nasal; i.p.: intraperitoneal; FCA: Freund Complete Adjuvant; IFA: Incomplete Freund Adjuvant; DDA: dimethyl dioctadecyl ammonium bromide; STP: 1\% pluronic 121, 10\% squalene, 0,4\%Tween 80; MPL-RIBI: Monophosphoryl Lipid A; BCG: bacille Calmette-Guérin; PLG: poly(lactide-co-glycolide); ASO: Aldicarb sulfone; Alum: aluminium hydroxide; QuilA: saponin; F: antigen selected based on presumed functional importance. I: antigen selected based on immune recognition. L: antigen selected based on location or accessibility to the immune system.

*Antigens tested in native form. 
Box 1. Advantages and disadvantages of different prokaryotic and eukaryotic expression systems

Advantages
Bacterial
- Ease of culture/rapid cell growth
- Minimum complexity of medium
- Large yields: up to $10 \%$ of mass
Yeast
- High density culture/rapid cell growth
- Minimum complexity of medium
- Secretion of proteins to medium
- Post-translational modifications present
Insect cells/Baculovirus
- Secretion of proteins to medium
- Proper folding of the proteins
- Simple glycosylations present, similar to mammalian
Mammalian cells
- Secretion of proteins to medium
- Proper folding of the proteins
- Complex glycosylations present
Disadvantages
Bacterial
- Insoluble proteins, difficult to recover/refold
- Post-translational modifications absent
- Yeast
- Refolding might be required
- High mannose glycosylation
- Cell growth slow
- Expensive medium
-

explained by the inappropriate folding of the peptide backbone and/or the lack of glycosylation on these bacterial recombinants. Many helminth antigens carry $N$ - and/or $O$ glycans on their peptide core, and some of these elicit strong immune responses [5-7]. An additional problem with $E$. coli is that much of the recombinant protein generated can end up in insoluble inclusion bodies. As a consequence, use of eukaryotic expression systems, such as yeast and baculovirus, has been proposed to address these problems with the bacterial recombinants [4].

However, there is little experimental evidence that the protective capacity of recombinant helminth antigens actually improves by switching from a bacterial to a eukaryotic expression system. Of the extensive list of recombinant antigens shown in Table 1, only three have been produced in different expression systems and subsequently tested for their protective capacities. The reduction in egg counts induced by vaccination with a cathepsin L3 from $F$. hepatica increased from $18 \%$ to $52 \%$ by changing from a yeastto a baculovirus-based expression system [8]. Recombinant versions of the fatty-acid-binding protein SjFABP of $S$. japonicum produced in $E$. coli and baculovirus induced similar levels of protection, that is $\sim 49 \%$ reduction in egg counts [9]. Finally, in the case of the $H$. contortus H11 antigen, the protective capacity actually fell using an enzymatically active baculovirus-expressed version compared with an E. coli recombinant [1].

Although the yeast and baculovirus expression systems clearly have the capability to glycosylate the recombinant antigens, this glycosylation can differ drastically from the helminth glycans. The yeast Saccharomyces cerevisiae, for example, can cover the peptide core with very large glycan trees, which potentially mask important peptide epitopes [4] or which can make the protein hyperantigenic [10]. This seemed to be the case with the yeast-expressed $F$. hepatica cathepsin L3, which induced less protection compared with the baculovirus-produced version, despite the fact that both recombinants induced comparable serological responses [8]. In addition, some glycan structures seem to be helminth specific and are not used by any other organism. In this context, the use of parasite cell lines and Caenorhabditis elegans as expression systems has been proposed [11-14].

\section{Importance of study of the native antigen}

In the past 5-10 years, vaccine research has focused mainly on producing and testing recombinants from different expression systems, without paying a lot of attention to the native proteins themselves. For most of the antigens listed in Table 1, there is no proof that the native proteins can actually induce a protective response. Apart from the cestode antigens, only ten vaccine candidates have been tested in native form, either purified or as part of a protective fraction (marked with an asterisk in Table 1). All the other antigens were selected on the basis of immune recognition, localization or presumed functional importance for the parasite (marked in Table 1 with I, L or F, respectively) and subsequently cloned for recombinant expression. If the recombinant versions of such proteins fail to induce protection in an animal trial, this could be because the recombinant was not produced in a correct form to induce a protective immune response, or because the protein itself is not a genuine protective antigen. In addition, even if prior knowledge is available that the native antigen is protective, it also seems rational to investigate what type of immune response needs to be induced and which peptide and/or glycan epitopes are involved in this before one can proceed to the expression of an 'immunologically active' recombinant. Much emphasis is currently put on the importance of glycan. However, although it is now well established that glycan residues can be extremely immunogenic, there is no scientific evidence (to our knowledge) that glycan epitopes are essential to induce protection in vivo.

So why are the native proteins not analysed properly? Possible reasons for this are timing and financial restrictions. It is, in many cases, difficult and time consuming to purify native helminth antigens. Some antigens, such as excretory-secretory products, are also scarce and very expensive to produce in terms of donor animals. There is also commercial pressure for fast results. Cloning and expressing an antigen is often quicker and cheaper than purifying the native version. Funding bodies are also more inclined to fund projects that are more 'high tech' and focus on 'new generation' vaccines based on recombinant DNA technology. However, it is questionable whether expressing 
and testing selected antigens, without testing and analysing the native protein, will be more cost effective in the long term.

In our opinion, more time and effort should be invested in the analysis of the native antigens. With the new generation of liquid chromatography equipment, it is now easier to separate and collect small amounts of protein. Mass spectrometry is also becoming a standard technique in reach for many research laboratories. In combination with the extensive parasite expressed sequence tag (EST) databases, they provide a powerful analytical platform. In contrast to 10 or 15 years ago, it is now possible to identify almost every component of a protective protein fraction in a matter of days or weeks. In addition, new methods for glycan analysis are currently leading to an explosion in information about the structures of parasite-derived glycans [5]. The use of these technologies, in combination with the advances in epitope identification by phage display, peptide libraries or bioinformatics [15], would give us crucial information on the antigens and thus help to make a more well-founded choice of expression system. In this context, new eukaryotic expression systems are emerging that can be engineered in their glycosylation pathway [16]. The use of such systems would avoid inappropriate glycosylation and if modified with helminth glycosyltransferases might theoretically produce a perfect copy of the native protein. Alternatively, if glycan proves to be essential for protection and impossible to produce in an expression system, the synthetic synthesis of carbohydrates might be a solution. This approach is currently being investigated in the search for vaccines against malaria and leishmaniasis [17].

\section{Concluding thoughts}

The lack of appropriate expression systems is often put forward as the big hurdle in the quest for recombinant parasite vaccines. However, instead of promoting and testing more 'high-tech' expression systems, we should consider the possibility that an in-depth analysis of the native antigen could speed up the development of recombinant vaccines in the long term. Of course, although in this opinion article we have focused solely on the importance of the antigen, it is essential to note that there are a number of additional parameters that are extremely important in vaccine development. The route of administration, the adjuvants, the use of model organisms, contaminating products from the expression system, antigen cocktails, the challenge infection and, finally, the method to assess the efficacy are also crucial aspects that should be taken into account. In addition, our expectations for a vaccine should be realistic. For some helminths, such as $O$. ostertagi and $F$. hepatica, it takes months to build up a natural protective immune response. Expecting that a vaccine should reach a higher level of protection in a shorter period is possibly too ambitious. For a detailed discussion of these factors, refer to Ref. [18].

\section{Acknowledgements}

This work was supported by a postdoctoral IWT grant ('Institute for the Promotion of Innovation by Science and Technology in Flanders') (OZM/ 040746) for V.D.M. and by a grant of 'Fund for Scientific ResearchFlanders' (FWO) (no. 3G007306). P.G. is a Postdoctoral Fellow of the
Fund for Scientific Research Flanders (Belgium) (F.W.O. - Vlaanderen) and the recipient of a Marie Curie reintegration grant (no. 028860). We apologize to authors of primary literature for failing to cite their work directly owing to reference-number limitations.

\section{References}

1 Smith, W.D. and Zarlenga, D.S. (2006) Developments and hurdles in generating vaccines for controlling helminth parasites of grazing ruminants. Vet. Parasitol. 139, 347-359

2 Lightowlers, M.W. (2006) Vaccines against cysticercosis and hydatidosis: Foundations in taeniid cestode immunology. Parasitol. Int. 55, S39-S43

3 Murray, L. et al. (2007) Expression and purification of an active cysteine protease of Haemonchus contortus using Caenorhabditis elegans. Int. J. Parasitol. 37, 1117-1125

4 Dalton, J.P. et al. (2003) Helminth vaccines: from mining genomic information for vaccine targets to systems used for protein expression. Int. J. Parasitol. 33, 621-640

5 Nyame, A.K. et al. (2004) Antigenic glycans in parasitic infections: implications for vaccines and diagnostics. Arch. Biochem. Biophys. 426, 182-200

6 Hokke, C.H. and Yazdanbakhsh, M. (2005) Schistosome glycans and innate immunity. Parasite Immunol. 27, 257-264

7 Gomez-Garcia, L. et al. (2005) Intact glycans from cestode antigens are involved in innate activation of myeloid suppressor cells. Parasite Immunol. 27, 395-405

8 Reszka, N. et al. (2005) Fasciola hepatica procathepsin L3 protein expressed by a baculovirus recombinant can partly protect rats against fasciolosis. Vaccine 23, 2987-2993

9 Zhong-Dao, W. et al. (2005) Development of a vaccine against Schistosoma japonicum in China: a review. Acta Trop. 96, 106-116

10 Cregg, J.M. et al. (1993) Recent advances in the expression of foreign genes in Pichia pastoris. Biotechnology (NY) 11, 905-910

11 Coyne, C.P. and Brake, D. (2001) Characterization of Haemonchus contortus derived cell populations propagated in vitro in a tissue culture environment and their potential to induce protective immunity in sheep. Int. J. Parasitol. 31, 359-376

12 Redmond, D.L. et al. (2001) Expression of Haemonchus contortus pepsinogen in Caenorhabditis elegans. Mol. Biochem. Parasitol. 112, $125-131$

13 Britton, C. and Murray, L. (2002) A cathepsin L protease essential for Caenorhabditis elegans embryogenesis is functionally conserved in parasitic nematodes. Mol. Biochem. Parasitol. 122, 21-33

14 Kwa, M.S.G. et al. (1995) Beta-tubulin genes from the parasitic nematode Haemonchus contortus modulate drug-resistance in Caenorhabditis elegans. J. Mol. Biol. 246, 500-510

15 De Groot, A.S. and Moise, L. (2007) Prediction of immunogenicity for therapeutic proteins: state of the art. Curr. Opin. Drug Discov. Devel. $10,332-340$

16 Brooks, S.A. (2006) Protein glycosylation in diverse cell systems: implications for modifications and analyses of recombinant proteins. Expert Rev. Proteomics 3, 345-359

17 Seeberger, P.H. and Werz, D.B. (2007) Synthesis and medical applications of oligosaccharides. Nature 446, 1046-1051

18 Vercruysse, J. and Claerebout, E. (2003) Assessment of the efficacy of helminth vaccines. J. Parasitol. 89 (Suppl.), S202-S209

19 Lightowlers, M.W. et al. (2003) Molecular and genetic characterisation of the host-protective oncosphere antigens of taeniid cestode parasites. Int. J. Parasitol. 33, 1207-1217

20 Plancarte, A. et al. (1999) Vaccination against Taenia solium cysticercosis in pigs using native and recombinant oncosphere antigens. Int. J. Parasitol. 29, 643-647

21 Hillyer, G.V. (2005) Faciola antigens as vaccines against fasciolosis and schistosomiasis. J. Helminthol. 79, 241-247

22 Ramos, C.R.R. et al. (2001) R-Sm14 - pRSETA efficacy in experimental animals. Mem. Inst. Oswaldo Cruz 96, 131-135

23 Gobert, G.N. and McManus, D.P. (2005) Update on paramysoin in parasitic worms. Parasitol. Int. 54, 101-107

24 Tran, M.H. et al. (2006) Tetraspanins on the surface of Schistosoma mansoni are protective antigens against schistosomiasis. Nat. Med. 12, 835-840

25 Pacifico, L.G. (2006) Immunization with Schistosoma mansoni $22,6 \mathrm{kDa}$ antigen induces partial protection against experimental 
infection in a recombinant protein form but not as DNA vaccine. Immunobiology 211, 97-104

26 Boulanger, D. et al. (1999) Schistosoma bovis: vaccine effects of a recombinant homologous glutathione S-transferase in sheep. Int. J. Parasitol. 29, 415-418

27 Boulanger, D. et al. (1994) Vaccination of goats against the trematode Schistosoma bovis with a recombinant homologous schistosomederived glutathione S-transferase. Parasite Immunol. 16, 399-406

28 Abáné, J.L. et al. (2000) Vaccination of mice against Schistosoma bovis with a recombinant fatty acid binding protein from Fasciola hepatica. Vet. Parasitol. 91, 33-42

29 Bungiro, R. and Cappello, M. (2004) Hookworm infection: new developments and prospects for control. Curr. Opin. Infect. Dis. 17, 421-426

30 Taylor, M.J. et al. (1996) Protective immunity induced by vaccination with Onchocerca volvulus tropomyosin in rodents. Parasite Immunol. $18,219-225$

31 Abraham, D. et al. (2001) Development of a recombinant antigen vaccine against infection with the filarial worm Onchocerca volvulus. Infect. Immun. 69, 262-270

32 MacDonald, A.J. et al. (2004) Ov-ASP-1, the Onchocerca volvulus homologue of the activation associated protein family is immunostimulatory and can induce protective anti-larval immunity. Parasite Immunol. 26, 53-62

$33 \mathrm{Wu}, \mathrm{Y}$. et al. (2004) The secreted larval acidic proteins (SLAPs) of Onchocerca spp. are encoded by orthologues of the alt gene family of Brugia malayi and have host protective potential. Mol. Biochem. Parasitol. 134, 213-224

34 Islam, M.K. et al. (2005) Vaccination with recombinant Ascaris suum 24-kDa antigen induces a Th1/Th2- mixed type immune resonse and confers high levels of protection against challenged Ascaris suum lungstage infection in BALB/c mice. Int. J. Parasitol. 35, 1023-1030

35 Tsuji, N. et al. (2001) Intranasal immunization with recombinant Ascaris suum 14-kilodalton antigen coupled with cholera toxin B subunit induces protective immunity to $A$. suum infection in mice. Infect. Immun. 69, 7285-7292
36 Tsuji, N. et al. (2003) Mice intranasally immunized with a recombinant 16-kilodalton antigen from roundworm ascaris parasites are protected against larval migration of Ascaris suum. Infect. Immun. 71, 53145323

37 Redmond, D.L. and Knox, D.P. (2004) Protection studies in sheep using affinity-purified and recombinant cysteine proteinases of adult Haemonchus contortus. Vaccine 22, 4252-4261

38 Redmond, D.L. and Knox, D.P. (2006) Further protection studies using recombinant forms of Haemonchus contortus cysteine proteinases. Parasite Immunol. 28, 213-219

39 Vercauteren, I. et al. (2004) Vaccination with an Ostertagia ostertagi polyprotein allergen protects calves against homologous challenge infection. Infect. Immun. 72, 2995-3001

40 Matthews, J.B. et al. (2001) Immunisation of cattle with recombinant acetylcholinesterase from Dictyocaulus viviparous and with adult worm ES products. Int. J. Parasitol. 31, 307-317

41 Hartmann, S. et al. (2006) A nematode allergen elicits protection against challenge infection under specific conditions. Vaccine 24, 3581-3590

42 Winter, J.A. et al. (2005) The assessment of hookworm calreticulin as a potential vaccine for necatoriasis. Parasite Immunol. 27, 139-146

43 Capron, A. et al. (2001) Vaccine strategies against schistosomiasis: From concepts to clinical trials. Int. Arch. Allergy Immunol. 124, 9-15

44 Boulanger, D. et al. (1999) Vaccine potential of a recombinant glutathione S-transferase cloned from Schistosoma haematobium in primates experimentally infected with an homologous challenge. Vaccine 17, 319-326

45 De Maere, V. et al. (2005) An aspartyl protease inhibitor of Ostertagia ostertagi: Molecular cloning, analysis of stage and tissue specific expression and vaccine trial. Mol. Biochem. Parasitol. 141, 81-88

46 De Maere, V. et al. (2005) Molecular analysis of astacin-like metalloproteases of Ostertagia ostertagi. Parasitology 130, 89-98

47 Vercauteren, I. et al. (2006) A small heat shock protein of Ostertagia ostertagi: stage-specific expression, heat inducibility and protection trial. J. Parasitol. 92, 1244-1250

\section{AGORA initiative provides free agriculture journals to developing countries}

The Health Internetwork Access to Research Initiative (HINARI) of the WHO has launched a new community scheme with the UN Food and Agriculture Organization.

As part of this enterprise, Elsevier has given hundreds of journals to Access to Global Online Research in Agriculture (AGORA). More than 100 institutions are now registered for the scheme, which aims to provide developing countries with free access to vital research that will ultimately help increase crop yields and encourage agricultural self-sufficiency.

According to the Africa University in Zimbabwe, AGORA has been welcomed by both students and staff. "It has brought a wealth of information to our fingertips", says Vimbai Hungwe. "The

information made available goes a long way in helping the learning, teaching and research activities within the University. Given the economic hardships we are going through, it couldn't have come at a better time."

For more information, visit www.aginternetwork.org 